\title{
Chemical evolution and stellar populations in the Sagittarius dwarf Spheroidal Galaxy
}

\author{
L. Sbordone ${ }^{1,2}$, P. Bonifacio ${ }^{1,2,3}$, G. Giuffrida ${ }^{4}$, G. Marconi ${ }^{5}$, \\ L. Monaco ${ }^{5}$ and S. Zaggia ${ }^{6}$ \\ ${ }^{1}$ CIFIST Marie Curie Excellence Team \\ ${ }^{2}$ Observatoire de Paris, GEPI 5, \\ place Jules Janssen, 92195 Meudon, France \\ email: luca.sbordone@obspm.fr \\ ${ }^{3}$ INAF - Osservatorio Astronomico di Trieste \\ ${ }^{4}$ Università di Roma "Tor Vergata" \\ ${ }^{5}$ ESO - European Southern Observatory - Chile \\ ${ }^{6}$ INAF - Osservatorio Astronomico di Padova
}

\begin{abstract}
The closest neighbour of the Milky Way (MW) the Sagittarius dwarf Spheroidal Galaxy (Sgr dSph) is being tidally destroyed by the interaction with our Galaxy, losing its stellar content along a huge stream clearly detectable within the Halo. This makes the Sgr dSph an ideal laboratory to study at the same time the chemical evolution of dwarf galaxies and their role in building bigger structures such as the MW. Since some years we are studying the stellar populations of the Sgr main body and stream, with particular attention to their detailed chemical composition. We collected detailed abundances (up to 22 elements, $\mathrm{O}$ to $\mathrm{Eu}$ ) for 27 stars in the Sgr dSph main body, 5 in the associated globular cluster Terzan 7, and 12 more in the trailing Sgr tidal arm (UVES@VLT and SARG@TNG data). We are also conducting a large FLAMES@VLT chemical and dynamical analysis aimed at obtaining metallicities, alpha-elements content and radial velocities from automated analysis of the spectra. Finally, we just completed the first large scale photometric and spectroscopic survey of the stellar populations across all the dSph main body extension with VIMOS@VLT, aimed at exploring the variations in stellar populations and at deriving radial velocity memberships for future high resolution spectroscopic analysis. The picture emerging from all these studies portraits a large and extremely complex object, with signs of a long and still unclear evolution. Metallicity varies across three orders of magnitude $([\mathrm{Fe} / \mathrm{H}]$ from -3 to 0 ), CMDs change surprisingly from the core to the outskirts of the galaxy, and the chemical composition of the most metal rich objects show a very characteristic signature, with underabundant alpha elements, deficient $\mathrm{Na}$, underabundant Fe-peak $\mathrm{Mn}, \mathrm{Co}, \mathrm{Ni}, \mathrm{Cu}$ and $\mathrm{Zn}$, and strongly enhanced n-capture elements La and Nd. This highly peculiar signature can also be effectively used to recognized stripped populations lost by Sgr in favour of the MW system, as clearly showed by the globular Palomar 12, which shows the same chemical anomalies detected in Sgr dSph.
\end{abstract}

Keywords. stars: abundances, stars: kinematics, galaxies: abundances, galaxies: dwarf, galaxies: individual: Sgr dSph, galaxies: stellar content, Galaxy: halo 